\title{
Avaliação da expansão rápida da maxila por meio da tomografia computadorizada: relato de um caso
}

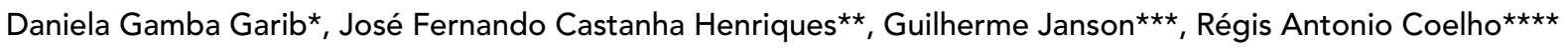

\begin{abstract}
Resumo
Este trabalho descreve os efeitos dentoesqueléticos e periodontais da expansão rápida da maxila (ERM), avaliados por meio da tomografia computadorizada (TC), em uma jovem de 11,6 anos de idade com má oclusão de Classe I e mordida cruzada posterior unilateral funcional. Durante a fase ativa da ERM, o expansor dentossuportado com parafuso Hyrax foi ativado $7 \mathrm{~mm}$. A paciente submeteu-se ao exame de TC helicoidal, antes da expansão e após a remoção do aparelho expansor, findo o período de três meses de contenção. Realizaram-se cortes axiais, de um milímetro de espessura, paralelamente ao plano palatino, englobando as regiões dentoalveolar e basal da maxila, até o terço inferior da cavidade nasal. Utilizando-se o recurso de reconstruções multiplanares, mensuraram-se pelo método computadorizado: as dimensões transversas maxilares, a inclinação dos dentes posteriores, a espessura das tábuas ósseas vestibular e lingual, e o nível da crista óssea alveolar vestibular. A expansão rápida da maxila ocasionou um significante aumento transverso em todas as regiões aferidas, com magnitude decrescente do arco dentário para a base óssea. Os dentes posteriores foram movimentados para vestibular, com um componente de inclinação e translação associados. Tal efeito ortodôntico ocasionou uma redução na espessura da tábua óssea vestibular, e um concomitante aumento na espessura da tábua óssea lingual. Após a expansão, observou-se o desenvolvimento de deiscências ósseas por vestibular dos dentes de ancoragem.
\end{abstract}

Palavras-chave: Expansão rápida da maxila. Tomografia computadorizada.

\section{INTRODUÇÃO}

O procedimento ortopédico de expansão rápida da maxila visa corrigir as atresias do arco dentário superior. Ao ocasionar a separação da sutura intermaxilar, aumenta o perímetro do arco den- tário $^{1}$, solucionando pequenos apinhamentos, ao mesmo tempo em que contribui para a compatibilização interarcos no plano transverso ${ }^{7,28}$.

Simultaneamente ao almejado efeito ortopédico, representado pela separação da sutura inter-

* Mestre e Doutora em Ortodontia pela Faculdade de Odontologia de Bauru - USP; Professora Associada da disciplina de Ortodontia da Universidade Cidade de São Paulo - UNICID; Coordenadora do curso de especialização em Ortodontia da Prevodonto Centro de Estudos - Rio de Janeiro.

** Professor Titular da disciplina de Ortodontia da Faculdade de Odontologia de Bauru - USP e coordenador do curso de pós-graduação em nível de Doutorado.

*** Professor Associado da disciplina de Ortodontia da Faculdade de Odontologia de Bauru - USP e coordenador do curso de pós-graduação em nível de Mestrado.

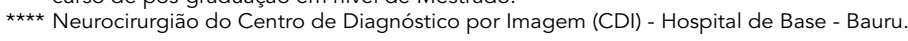


maxilar, a expansão rápida da maxila inevitavelmente também ocasiona um efeito ortodôntico, quando movimenta os dentes posteriores para vestibular $1,5,7,10,20,21,23,24,25,36,38,40,42,52$. A força liberada pelo expansor promove áreas de compressão no ligamento periodontal dos dentes sobre o qual se apóia ${ }^{42}$. Posteriormente, a reabsorção do osso alveolar vestibular redunda em movimento dentário para esta direção $0^{42}$. Alguns estudos demonstraram uma forte correlação entre o movimento dentário para vestibular e o desenvolvimento de deiscências ósseas, mediante a utilização de aparelhos ortodônticos em animais ${ }^{43,45,51}$. Transportando a mesma preocupação para a expansão rápida da maxila, a busca literária mostrou escassos trabalhos sobre as condições ósseas periodontais após este procedimento ${ }^{19,42}$, principalmente em humanos ${ }^{19}$. Tal deficiência justifica-se. As radiografias convencionais que compõem a documentação ortodôntica, mostrando apenas imagens bidimensionais e sobrepostas, não evidenciam a espessura e o nível das tábuas ósseas vestibular e lingual ${ }^{17,18,46}$. Como avaliar estas regiões em seres humanos, considerando-se que os estudos histológicos são inviáveis, e o rebatimento de um retalho poderia trazer conseqüências periodontais irreversíveis, esbarrando no senso ético das pesquisas?

A premente necessidade de definir as conseqüências periodontais da ERM, realizada com muita freqüência na clínica diária, evocou um novo método de diagnóstico: a tomografia computadorizada (TC). Reproduzindo uma verdadeira secção dos maxilares, nos três planos do espaço ${ }^{15,16,22,26,30,31}$, a TC evidencia a imagem das tábuas ósseas vestibular e lingual, ao mesmo tempo em que permite mensurar as dimensões transversas em quaisquer região da maxila. Alguns autores aplicaram esta modalidade de diagnóstico por imagem na Ortodontia $^{12,13,14,33,37,48}$. Porém, deve ficar claro que não se propõe a TC como parte da documentação ortodôntica. Principalmente devido à maior quantidade de radiação emitida ${ }^{11,27,47}$ e secundariamente ao preço elevado, indica-se a TC na Odontologia apenas quando compensar a relação custo-benefício ${ }^{15,33}$. Atualmente, as principais indicações restringem-se aos casos de fraturas complexas da face $^{6,30,31,44}$, nas anomalias craniofaciais ${ }^{26}$, no diagnóstico e acompanhamento longitudinal de patologias dos maxilares $6,8,9,16,26,34,35$, no planejamento de múltiplos implantes ${ }^{15,31}$, e para a aferição dos componentes esqueléticos da $\mathrm{ATM}^{16,31}$.

O presente trabalho visou avaliar, por meio da tomografia computadorizada, os efeitos dentoesqueléticos e periodontais da expansão rápida da maxila em uma jovem na fase de dentadura permanente. $\mathrm{O}$ caso descrito pertence a uma amostra de pesquisa da Faculdade de Odontologia de Bauru, Universidade de São Paulo, voltada para a avaliação e comparação dos efeitos ortopédicos, ortodônticos e periodontais dos expansores dentomucossuportado e dentossuportado, sob a ótica da TC.

\section{DIAGNÓSTICO INICIAL}

A paciente C.P.N., brasileira, gênero feminino, 11,6 anos de idade, procurou por tratamento ortodôntico na Faculdade de Odontologia de Bauru, Universidade de São Paulo, queixando-se do canino superior direito mal posicionado. $\mathrm{Na}$ análise facial frontal, apresentava um padrão mesofacial com selamento labial passivo, e na análise lateral, demonstrava um perfil reto e aspecto estético harmonioso (Fig. 1A, B). Ao exame intrabucal, a paciente encontrava-se na dentadura permanente jovem mostrando uma má oclusão de Classe I com mordida cruzada unilateral funcional do lado direito, o incisivo lateral superior direito e o segundo pré-molar superior esquerdo também estavam em mordida cruzada, e o canino superior direito em suave infra-vestíbulo-versão (Fig. 1C-1G). No momento do diagnóstico, constatou-se um padrão funcional normal quanto à respiração e à deglutição, mas havia o histórico de uso prolongado de mamadeira, além de um hábito persistente de onicofagia. A análise morfológica da telerradiografia confirmava uma padrão facial favorável (Fig. 2). 

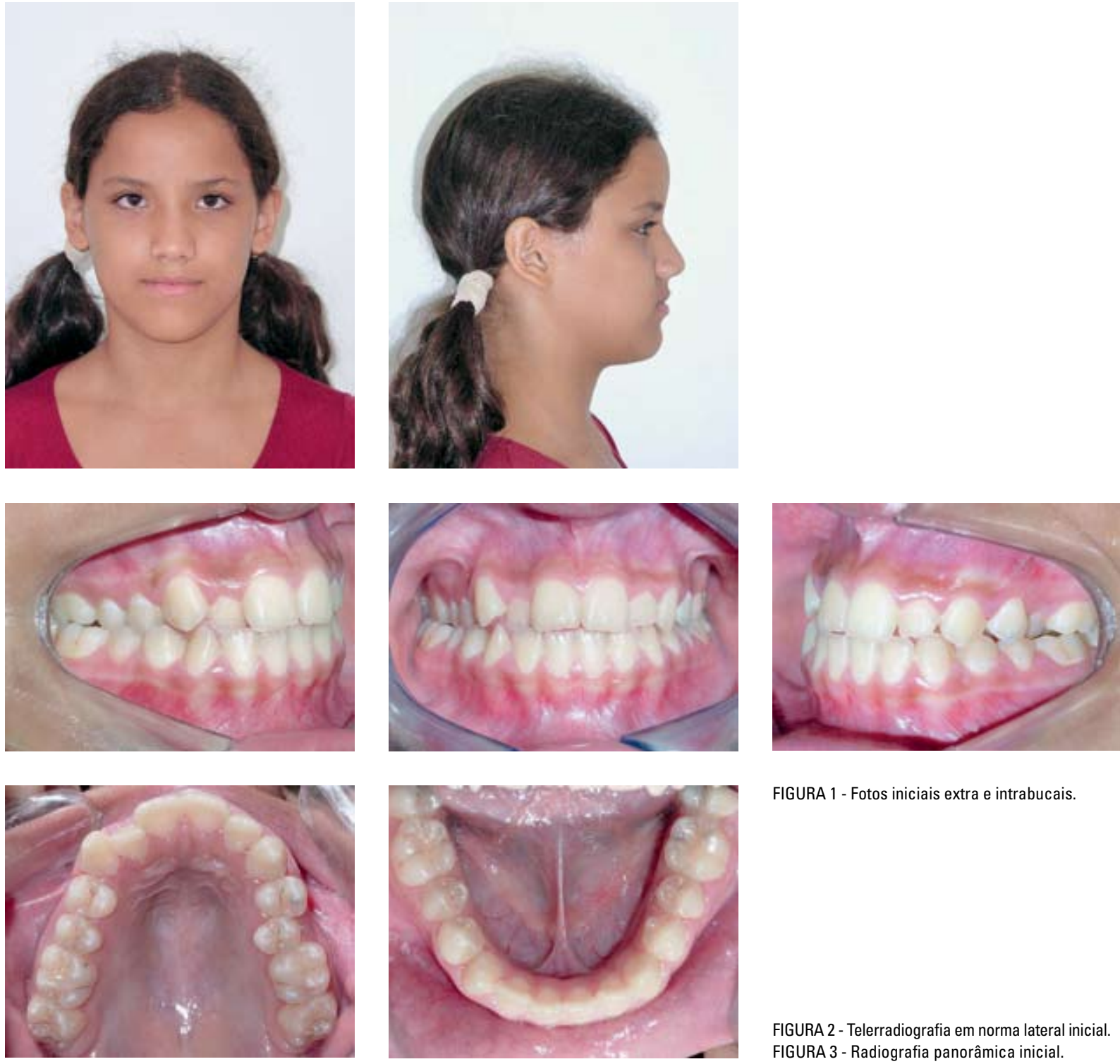

FIGURA 1 - Fotos iniciais extra e intrabucais.

FIGURA 2 - Telerradiografia em norma lateral inicial. FIGURA 3 - Radiografia panorâmica inicial.
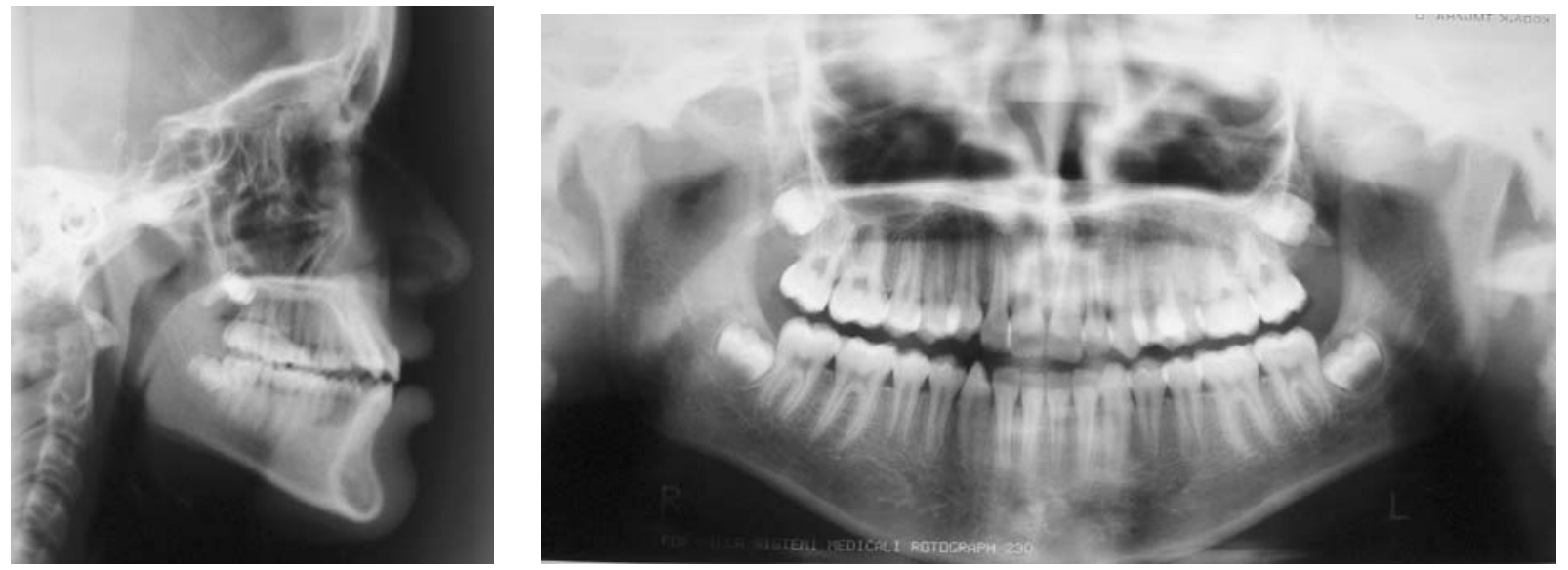


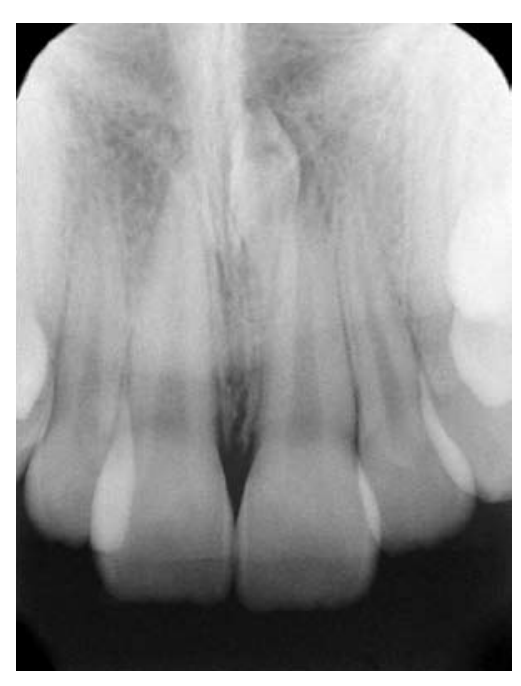

FIGURA 4 - Radiografia periapical dos incisivos superiores mostrando o supranumerário mesiodens.

FIGURA 5 - Reconstrução sagital em tomografia computadorizada, passando pelo centro do mesiodens.

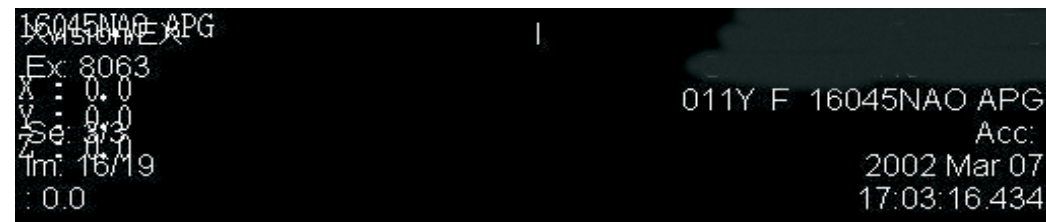

\section{$512 \times 512$}
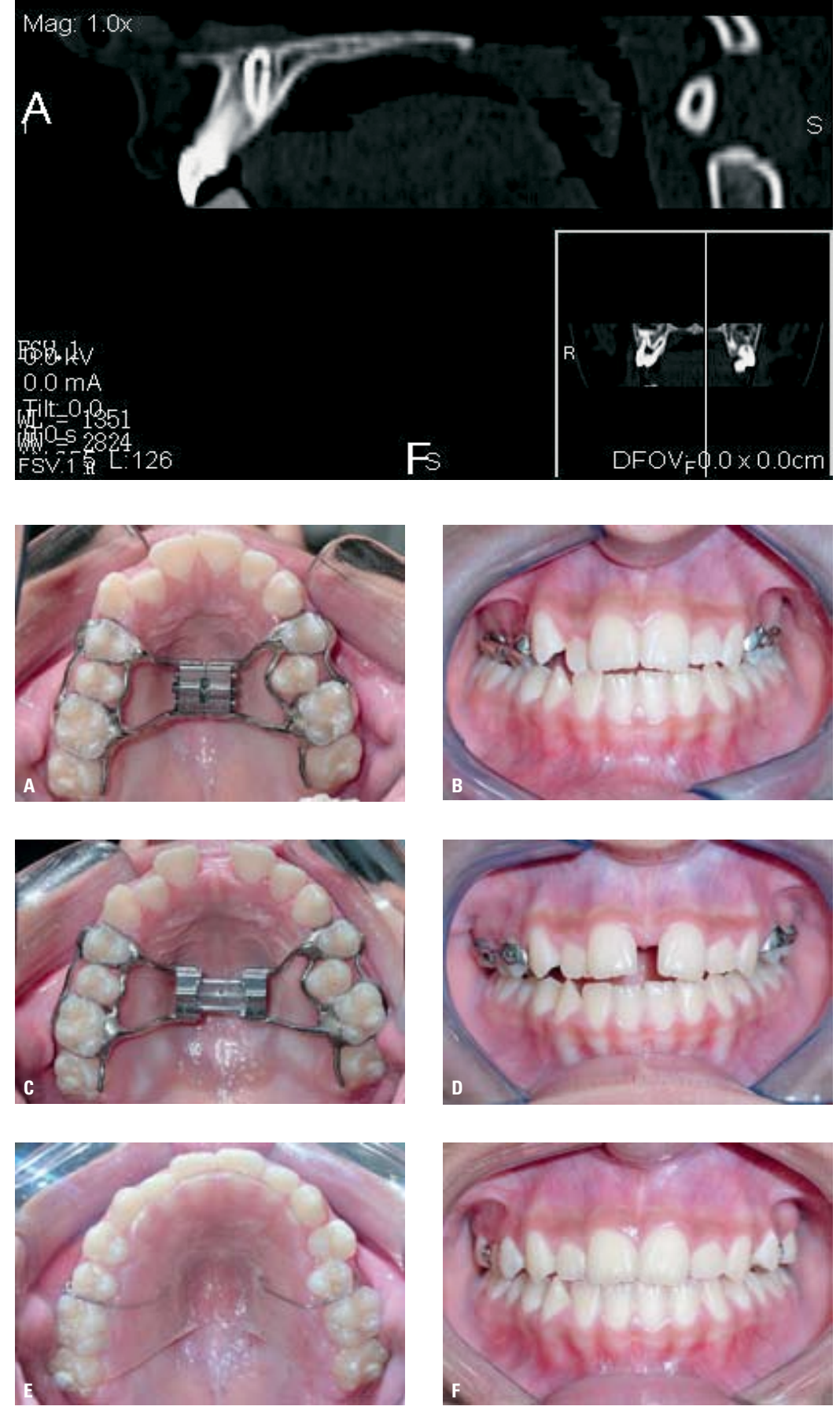

FIGURA 6 - A, B) Fotos pré-expansão; C, D) Fotos pós-expansão; E, F) Fotos pós-contenção. 


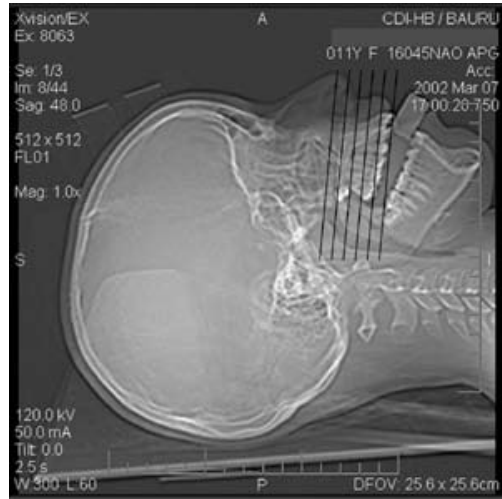

FIGURA 7 - Escanograma com a representação da área maxilar englobada no exame de tomografia computadorizada.

FIGURA 8 - Reconstrução multiplanar. No quadrante inferior esquerdo visualiza-se o corte original axial. Nos quadrantes superiores esquerdo e direito, e no quadrante inferior direito aparecem, res pectivamente, as reconstruções coronal, sagital e oblíqua (neste caso ortorradial).

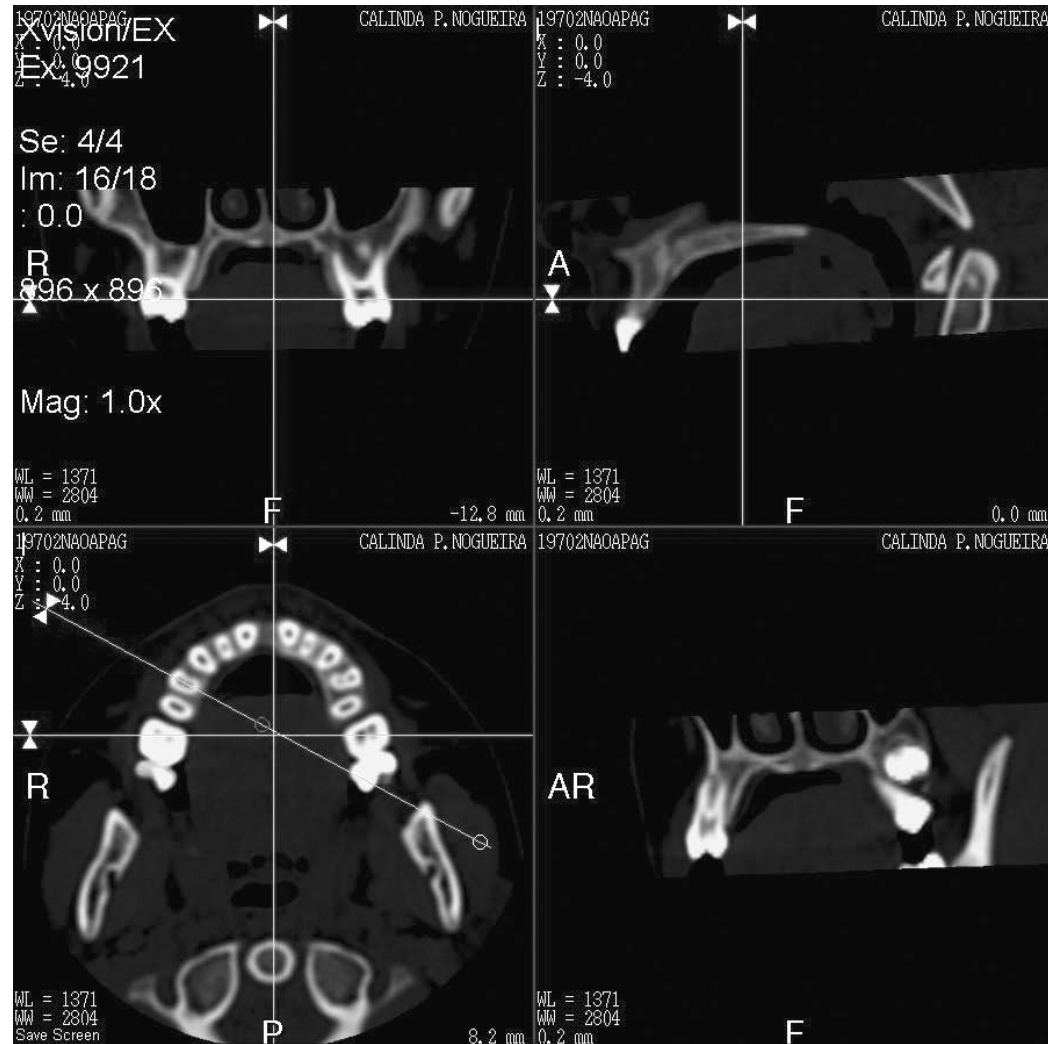

Screen

D
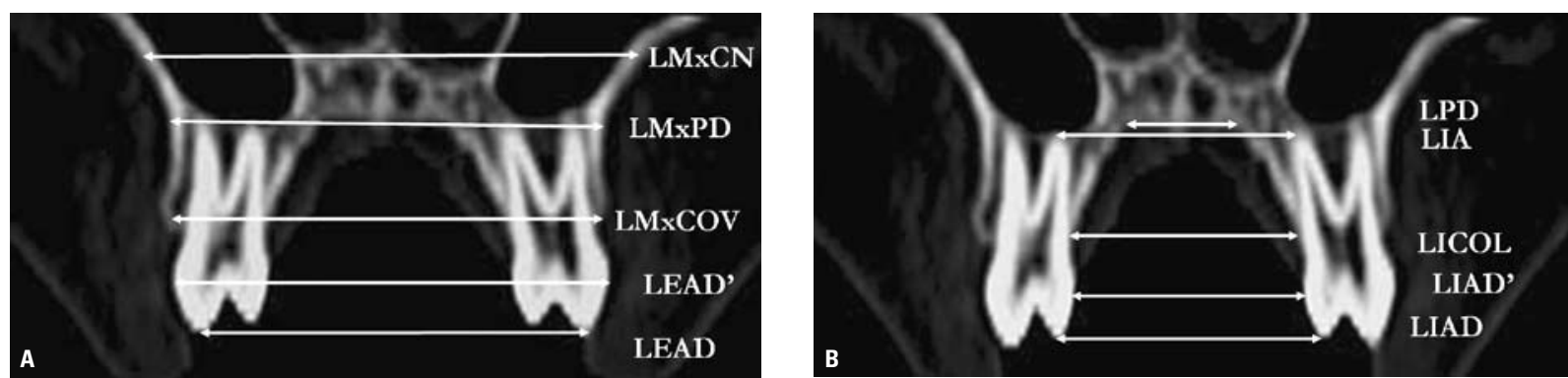

FIGURA 9 - A) Dimensões transversas externas da maxila; B) Dimensões transversas internas da maxila.
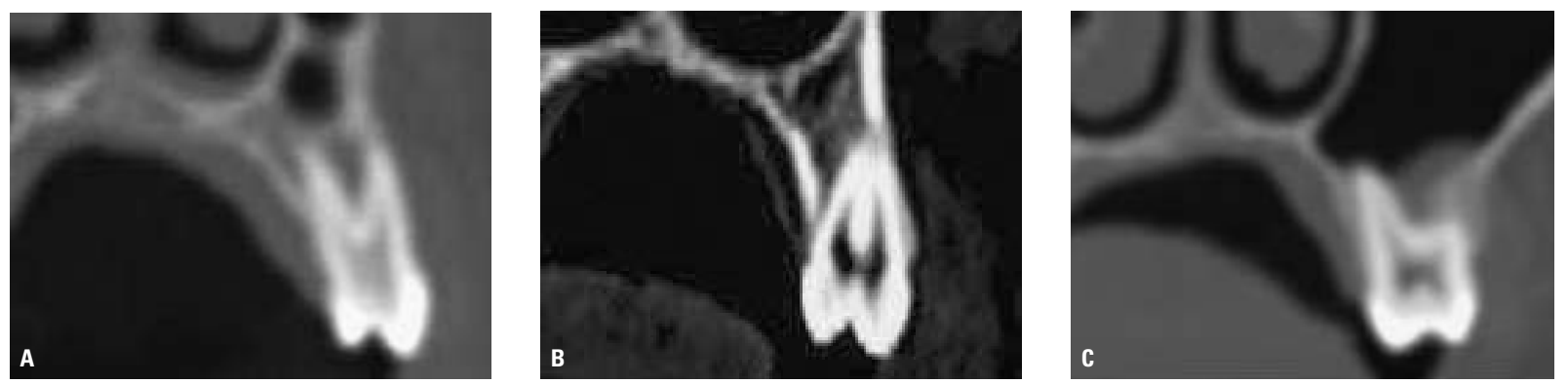

FIGURA 10 - Reconstruções ortorradiais passando pelo centro da raiz palatina do A) primeiro pré-molar; B) segundo pré-molar e C) primeiro molar. 


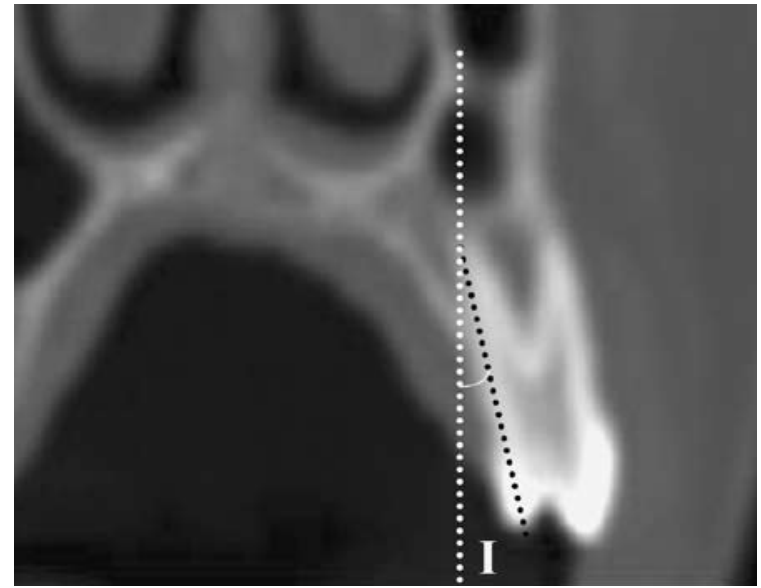

FIGURA 11 - Mensuração da inclinação axial dos dentes posteriores.

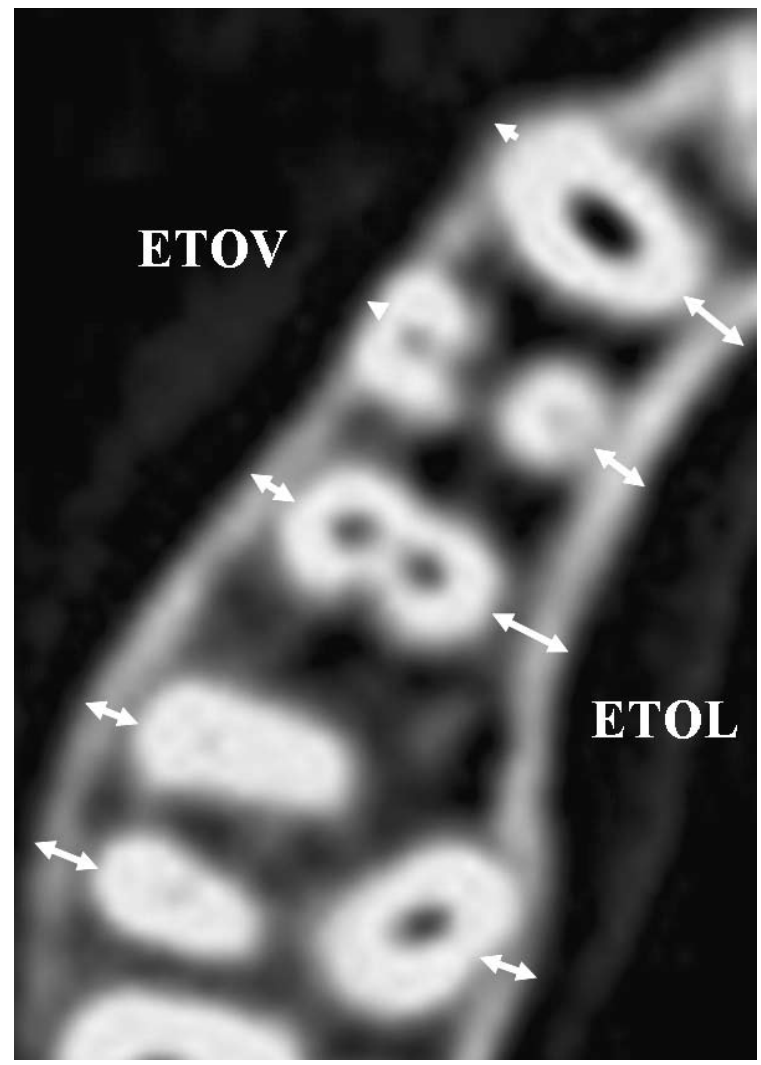

FIGURA 13 - Ampliação da área de interesse e mensuração da espessura das tábuas ósseas.

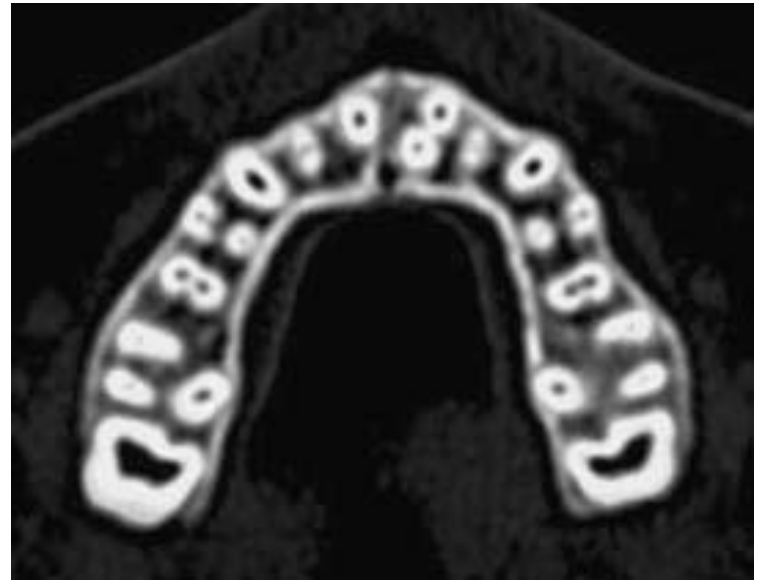

FIGURA 12 - Corte axial ao nível da trifurcação do primeiro molar superior direito.

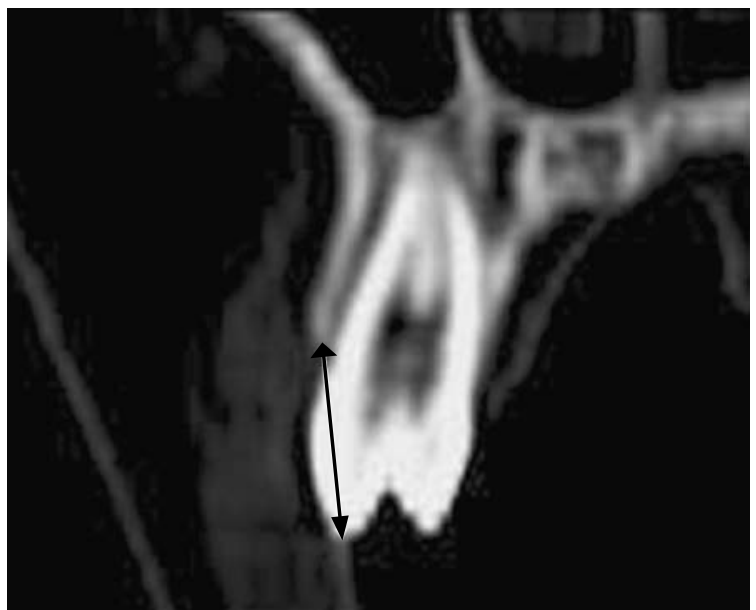

FIGURA 14 - Mensuração do nível da crista óssea alveolar vestibular.

Na radiografia panorâmica (Fig. 3), vislumbravamse as estruturas ósseas preservadas, todos os dentes permanentes irrompidos, com exceção dos terceiros molares, e a presença de um supranumerário mesiodens, visualizado com mais nitidez na radiografia periapical dos incisivos superiores (Fig. 4).

Previamente ao tratamento ortodôntico, a jovem foi encaminhada para tratamento odontológico geral e para a remoção cirúrgica do dente supranumerário. No entanto, considerando a posição desfavorável do mesiodens para o acesso cirúrgico (Fig. 5), o profissional decidiu-se pela manutenção e proservação radiográfica longitudinal do supranumerário. 

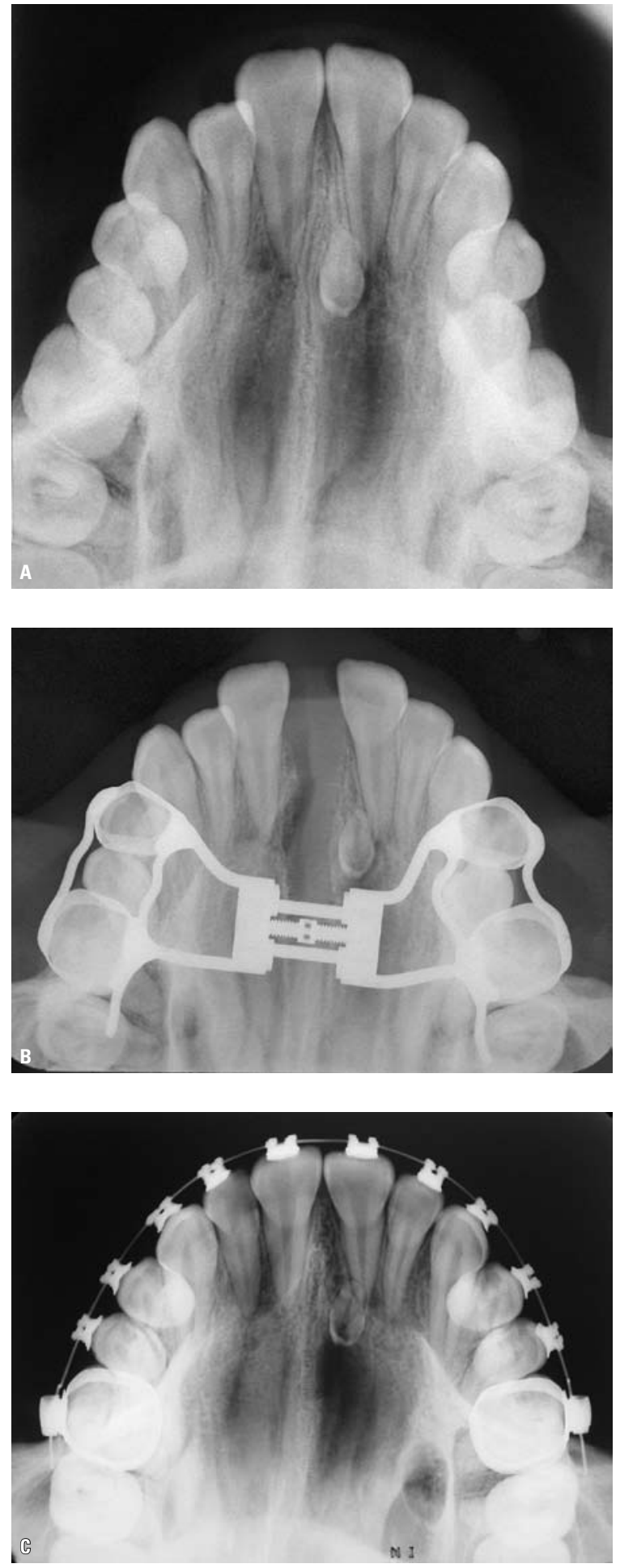

FIGURA 15 - Radiografias oclusais A) pré-expansão; B) pós-expansão e C) pós-contenção, demonstrando o comportamento do supranumerário mesiodens.

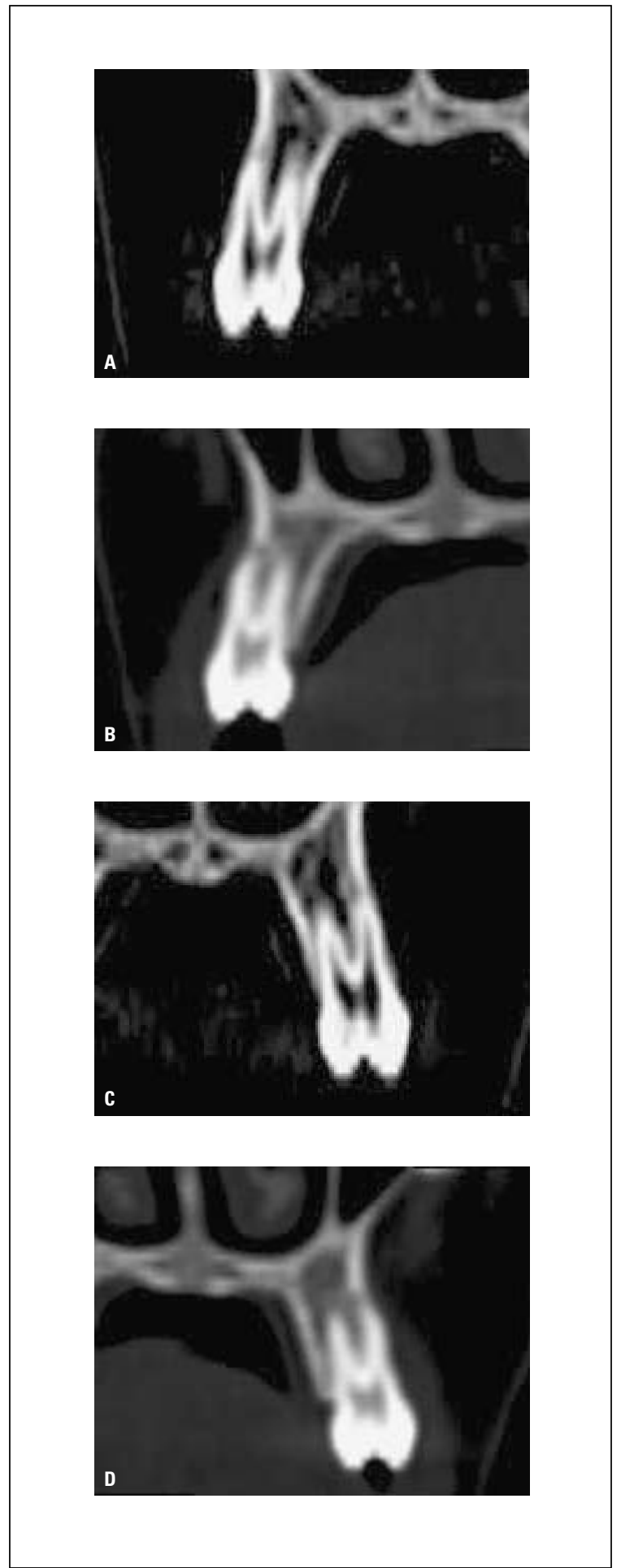

FIGURA 16 - Reconstruções ortorradiais passando pelo centro da coroa dos primeiros pré-molares superiores direito e esquerdo $\mathbf{A}, \mathbf{C}$ ) antes da ERM e B, D) após a remoção do aparelho expansor. Notar o desenvolvimento de deiscências ósseas após a expansão. 
O planejamento ortodôntico incluiu a expansão rápida da maxila para correção da mordida cruzada posterior, seguida pela mecanoterapia corretiva com aparelho fixo pré-ajustado.

\section{A EXPANSÃO RÁPIDA DA MAXILA}

A jovem submeteu-se ao procedimento de expansão rápida da maxila, previamente à mecanoterapia corretiva, utilizando-se o expansor dentossuportado, com parafuso Hyrax (Fig. 6A, B). $\mathrm{O}$ parafuso de $7 \mathrm{~mm}$, da marca Dentaurum (Dentaurum, Ispringen, Alemanha), foi ativado com uma volta completa no dia seguinte à instalação do aparelho, e com $1 / 4$ de volta de manhã e $1 / 4$ à tarde nos demais dias ${ }^{20}$, até o seu travamento ocorrido no décimo quinto dia. Após a fase ativa da expansão, fixou-se o parafuso com resina acrílica (Fig. 6C, D) e o aparelho somente foi removido após um período de três meses de contenção (Fig. 6E, F).

\section{O EXAME DE TOMOGRAFIA COMPUTADO- RIZADA E AS MENSURAÇÕES}

Realizou-se o exame de tomografia computadorizada antes da expansão, e após a remoção do expansor, findo o período de três meses de contenção. Para tanto, foi empregado o aparelho de tomografia computadorizada helicoidal Toshiba, modelo Xvision EX (Toshiba Corporation Medical Systems Company, Otawara-Shi, Japão), trabalhando com $120 \mathrm{kV}, 100 \mathrm{~mA}$ e tempo de exposição de um segundo por corte. Utilizou-se o filtro 30 para melhor evidenciação dos tecidos duros. A dimensão da matriz equivaleu a 512 X 512 pixels, com campo de visão (FOV) de 12,6 X 12,6cm. Foram realizados cortes axiais, de um milímetro de espessura, paralelamente ao plano palatino, englobando as regiões dentoalveolar e basal da maxila, até o terço inferior da cavidade nasal (Fig. 7). A extensão escaneada totalizou $40 \mathrm{~mm}$, e portanto, 40 cortes.

Posteriormente, os dados foram enviados a uma Workstation (Silicon Graphics, Toshiba Corporation Medical Systems Company, Otawara-Shi,
Japão) onde o software Alatoview (Toshiba Corporation Medical Systems Company, OtawaraShi, Japão) possibilitou a execução das reconstruções multiplanares (Fig. 8) e das mensurações pelo método computadorizado, como descrito a seguir.

\section{Dimensões transversas maxilares}

Para mensurar as dimensões transversas da maxila, utilizaram-se duas reconstruções coronais, perpendiculares ao plano sagital mediano. A primeira passando pelo centro da raiz palatina do primeiro pré-molar direito e a segunda coincidindo com o centro da raiz palatina do primeiro molar direito. Em cada uma destas duas imagens, nas fases pré e pós-expansão, obtiveram-se as seguintes grandezas lineares (Fig. 9A, B):

- LMxCN: largura da maxila ao nível do assoalho da cavidade nasal;

- LMxPD: largura da maxila ao nível do palato duro;

- LMxCOV: largura da maxila ao nível da crista óssea alveolar vestibular;

- LEAD: largura externa do arco dentário, mensurada ao nível das pontas de cúspide vestibulares;

- LEAD': largura externa do arco dentário, mensurada ao nível da região mais proeminente do contorno da face vestibular dos dentes posteriores;

- LPD: largura do palato duro;

- LIA: largura interápices, mensurada na raiz palatina dos dentes posteriores;

- LICOL: largura intercristas ósseas alveolares linguais;

- LIAD: largura interna do arco dentário, mensurada ao nível das pontas de cúspide palatinas;

- LIAD': largura interna do arco dentário, mensurada ao nível da região mais proeminente do contorno da face lingual dos dentes posteriores;

\section{Inclinação dos dentes posteriores}

Com vistas a analisar a inclinação axial dos dentes póstero-superiores, utilizaram-se reconstruções oblíquas ortorradiais, perpendiculares ao contorno do arco dentário, passando pelo 
centro da raiz palatina dos primeiros pré-molares (Fig. 10A), pelo centro da raiz dos segundos prémolares (Fig. 10B), e pelo centro da raiz palatina dos primeiros molares (Fig. 10C), de ambos os lados. Em cada uma dessas imagens, obteve-se a seguinte grandeza angular (Fig. 11):

- I: inclinação dentária, correspondente ao ângulo formado por uma linha passando pela ponta de cúspide palatina e ápice da raiz palatina, em relação a uma perpendicular à borda inferior da imagem. À inclinação vestibular foi atribuído valor positivo e à inclinação lingual, valor negativo.

\section{Espessura das tábuas ósseas vestibular e lin- gual}

Para mensurar a espessura do osso alveolar que envolve os dentes póstero-superiores por vestibular e lingual foi utilizado um corte axial, paralelo ao plano palatino, ao nível da trifurcação do primeiro molar superior direito (Fig. 12). Neste corte axial, empregando-se ampliação da imagem (Fig. 13), vislumbraram-se:

- ETOV: espessura da tábua óssea vestibular, mensurada desde o limite externo da cortical vestibular até o perímetro vestibular da raiz dentária, no centro do canino, primeiro pré-molar, segundo pré-molar e centro das raízes mésio e distovestibular do primeiro molar, de ambos os lados;

- ETOL: espessura da tábua óssea lingual, mensurada desde o limite externo da cortical palatina até o perímetro palatino da raiz dentária, no centro do canino, primeiro pré-molar, segundo prémolar e centro da raiz palatina do primeiro molar, de ambos os lados.

Diante de giroversões dentárias, mensurou-se a espessura da tábua óssea na região em que a raiz mais se aproximava do contorno externo do rebordo alveolar.

\section{Nível da crista óssea vestibular}

A fim de avaliar o nível da crista óssea vestibular dos dentes póstero-superiores, utilizaram-se reconstruções ortorradiais, perpendiculares ao con- torno do arco dentário, passando pelo centro da face vestibular dos caninos e pré-molares, e pelo centro, mesial e distal da face vestibular dos primeiros molares. Em cada uma dessas reconstruções, mensurou-se a seguinte grandeza linear (Fig. 14):

- NCOV: nível da crista óssea vestibular, estabelecido pela distância entre a ponta de cúspide vestibular e a crista óssea alveolar vestibular.

\section{RESULTADOS DA AVALIAÇÃO QUANTITATI- VA DAS IMAGENS EM TC}

Antes de iniciarmos uma análise numérica dos efeitos da ERM, expressos na tomografia computadorizada, vale observar o comportamento do mesiodens durante a expansão, na seqüência de radiografias oclusais (Fig. 15A, B, C). O dente supranumerário acompanhou o movimento lateral da hemimaxila esquerda. Após o período de contenção, com o restabelecimento da sutura intermaxilar, o mesiodens retornou à sua posição inicial.

Os valores pré e pós-expansão das mensurações aferidas na TC estão expostos nas tabelas 1 a 4. Ao final do nome de cada medida, após um hífen, estará indicado o número do dente onde a mensuração foi realizada. Como exemplo, a medida LMxPD-4 corresponde à largura da maxila ao nível do palato duro, na região dos primeiros pré-molares. A medida I-6 equivale à inclinação axial dos primeiros molares superiores, traduzindo a média entre os lados direito e esquerdo. Nos primeiros molares permanentes, algumas mensurações foram realizadas na região mesiovestibular, mediana e distovestibular, representadas respectivamente por $\mathrm{M}, \mathrm{Md}$ e $\mathrm{D}$.

A expansão rápida da maxila mostrou muita eficiência em aumentar as dimensões transversas maxilares, em todas as áreas aferidas, com efeito decrescente em direção superior (Tab. 1). O arco dentário demonstrou os maiores incrementos em largura, muito próximos à quantidade de ativação do parafuso $(7 \mathrm{~mm})$, tanto na região dos pré-molares como na dos molares. $\mathrm{O}$ aumento da largura externa do arco dentário (LEAD), mensurada ao 
nível das pontas de cúspide vestibulares, excedeu a expansão do parafuso, provavelmente devido à adição do efeito de inclinação dentária. Os menores aumentos transversos da maxila foram registrados ao nível do assoalho da cavidade nasal (LMxCN). Nesta região, o efeito ortopédico expressou, em média, $50 \%$ da abertura total do aparelho expansor. As alterações em largura ao nível do palato duro (LMxPD e LPD) e das cristas ósseas alveolares (LMxCOV e LICOL) exibiram magnitudes intermediárias. Esta gradação diferencial no efeito da ERM explica-se pela rotação lateral das hemimaxilas, com fulcro localizado próximo à sutura frontomaxilar, somada à ocorrência do efeito ortodôntico, representado pelo movimento vestibular dos dentes posteriores $1,5,7,10,20,21,23,24,25,36,38,40,42,52$.

O procedimento de ERM inclinou os dentes

Tabela 1 - Alterações (em milímetros) nas dimensões transversas maxilares, na região dos primeiros pré-molares e dos primeiros molares.

\begin{tabular}{|c|c|c|c|}
\hline Medidas & $\begin{array}{c}\text { Pré- } \\
\text { expansão }\end{array}$ & $\begin{array}{c}\text { Pós- } \\
\text { expansão }\end{array}$ & Alteração \\
\hline LMxCN-4 & 36 & 39,3 & 3,3 \\
\hline LMxPD-4 & 36,7 & 42,2 & 5,5 \\
\hline LMxCOV-4 & 42,2 & 48,6 & 6,4 \\
\hline LEAD-4 & 40,2 & 48,6 & 8,4 \\
\hline LEAD'-4 & 42,3 & 50,6 & 8,3 \\
\hline LIA-4 & 25,9 & 32 & 6,1 \\
\hline LPD-4 & 14 & 17,1 & 3,1 \\
\hline LICOL-4 & 24,8 & 29,3 & 4,5 \\
\hline LIAD-4 & 29,2 & 36,6 & 7,4 \\
\hline LIAD'-4 & 24,1 & 30,4 & 6,3 \\
\hline LMxCN-6 & 69,2 & 73,2 & 4,0 \\
\hline LMxPD-6 & 61,5 & 66,5 & 5,0 \\
\hline LMxCOV-6 & 51 & 58,4 & 7,4 \\
\hline LEAD-6 & 47,4 & 57,0 & 9,5 \\
\hline LEAD'-6 & 52,3 & 61,6 & 9,3 \\
\hline LIA-6 & 27,9 & 34,4 & 6,5 \\
\hline LPD-6 & 19,7 & 21,6 & 2,0 \\
\hline LICOL-6 & 30,4 & 34,9 & 4,5 \\
\hline LIAD-6 & 37,5 & 45,0 & 7,4 \\
\hline LIAD'-6 & 31 & 37,3 & 6,3 \\
\hline
\end{tabular}

posteriores para vestibular (Tab. 2). Porém, a magnitude da inclinação dos segundos pré-molares revelou-se maior que a dos dentes de ancoragem direta, primeiros pré-molares e primeiros molares. Esse diferencial na quantidade de inclinação pode correlacionar-se ao modo de transmissão da força expansiva. Os segundos pré-molares receberam a força de expansão por meio do contato do fio de conexão lingual com a sua face homônima. Com uma força simples aplicada na coroa, distante do centro de resistência, criou-se um momento para

Tabela 2 - Alterações (em graus) nas inclinações dos dentes posteriores.

\begin{tabular}{|c|c|c|c|}
\hline Medidas & $\begin{array}{c}\text { Pré- } \\
\text { expansão }\end{array}$ & $\begin{array}{c}\text { Pós- } \\
\text { expansão }\end{array}$ & Alteração \\
\hline I-4 & 5,1 & 6,4 & 1,3 \\
\hline I-5 & $-8,4$ & $-4,1$ & 4,3 \\
\hline I-6 & 12,7 & 15,5 & 2,8 \\
\hline
\end{tabular}

Tabela 3 - Alterações (em milímetros) na espessura das tábuas ósseas vestibular e lingual.

\begin{tabular}{|c|c|c|c|}
\hline Medidas & $\begin{array}{c}\text { Pré- } \\
\text { expansão }\end{array}$ & $\begin{array}{c}\text { Pós- } \\
\text { expansão }\end{array}$ & Alteração \\
\hline ETOV-3 & 0,8 & 0,9 & 0,1 \\
\hline ETOV-4 & 0,7 & 0,0 & $-0,7$ \\
\hline ETOV-5 & 1,5 & 1,3 & $-0,2$ \\
\hline ETOV-6M & 1,4 & 0,3 & $-1,1$ \\
\hline ETOV-6D & 2,0 & 0,9 & $-1,1$ \\
\hline ETOL-3 & 2,2 & 2,1 & $-0,1$ \\
\hline ETOL-4 & 1,8 & 3,1 & 1,3 \\
\hline ETOL-5 & 1,9 & 2,3 & 0,4 \\
\hline ETOL-6 & 1,2 & 2,9 & 1,7 \\
\hline
\end{tabular}

Tabela 4 - Alterações (em milímetros) no nível da crista óssea vestibular.

\begin{tabular}{|c|c|c|c|}
\hline Medidas & $\begin{array}{c}\text { Pré- } \\
\text { expansão }\end{array}$ & $\begin{array}{c}\text { Pós- } \\
\text { expansão }\end{array}$ & Alteração \\
\hline NCOV-3 & 12,4 & 11,1 & $-1,3$ \\
\hline NCOV-4 & 10,3 & 18,7 & 8,5 \\
\hline NCOV-5 & 9,0 & 8,9 & 0 \\
\hline NCOV-6M & 8,7 & 11,1 & 2,4 \\
\hline NCOV-6Md & 8,0 & 9,3 & 1,3 \\
\hline NCOV-6D & 8,9 & 9,2 & 0,3 \\
\hline
\end{tabular}


vestibular, culminando em algum componente de inclinação neste sentido ${ }^{41}$. Por outro lado, os dentes de ancoragem receberam a força expansiva através de bandas rigidamente fixadas ao corpo do aparelho. À medida que o parafuso foi ativado, as bandas, largas no sentido cervico-oclusal, ofereceram resistência à tendência de inclinação, movimentando os dentes de ancoragem predominantemente de corpo para vestibular. Pode-se admitir tal explicação ao analisar, na tabela 1 , as alterações na largura interápices (LIA) dos primeiros pré-molares e primeiros molares. O aumento de aproximadamente $6 \mathrm{~mm}$ nessa dimensão quase se igualou à quantidade de ativação do expansor $(7 \mathrm{~mm})$.

O efeito ortodôntico da expansão rápida da maxila, representado pelo movimento vestibular dos dentes posteriores, e comentado anteriormente, estimulou uma redução na espessura da tábua óssea vestibular (ETOV). A tabela 3 mostra a ocorrência de uma redução na espessura do osso alveolar por vestibular dos dentes que receberam as bandas, enquanto nos dentes vizinhos, caninos e segundos pré-molares, a tábua óssea permaneceu praticamente inalterada. A redução na espessura da tábua óssea vestibular sinalizou a ausência de equivalente aposição óssea compensatória sob o periósteo vestibular, pelo menos durante o intervalo de quatro meses, entre o início das ativações e a remoção do aparelho expansor.

Contrariamente à tábua óssea vestibular, a espessura do osso alveolar lingual aumentou com a expansão, principalmente na região dos dentes de ancoragem (Tab. 3).

Após a ERM, constatou-se o desenvolvimento de deiscências ósseas por vestibular dos dentes de ancoragem, traduzidas pelo aumento da dimensão NCOV (Tab. 4). Os primeiros pré-molares exibiram as maiores reabsorções da crista óssea vestibular (Fig. 16). Nos primeiros molares, a região mesiovestibular, mais proeminente e, portanto, recoberta inicialmente por uma tábua óssea delgada, mostrou maiores alterações que as regiões média e distovestibular. Os segundos pré-molares, que não receberam bandas, e os caninos, não envolvidos pelo expansor, não revelaram redução no nível da crista óssea alveolar.

Clinicamente, nesta jovem não se observaram recessões gengivais imediatamente após a expansão. Já se reconhece como os principais fatores predisponentes às recessões gengivais, os dentes posicionados ou movimentados para vestibular, as deiscências ósseas e a presença de mucosa ceratinizada fina e friável $1^{2,3,4,32,34,49,50,53}$. Contudo, as recessões apenas são desencadeadas diante do trauma mecânico da escovação ou da inflamação placa-induzida ${ }^{34,50}$. Portanto, a qualidade da mucosa ceratinizada e principalmente, o modo de escovação devem ser rigorosamente controlados em pacientes submetidos à ERM. Na ausência dos fatores desencadeantes, mesmo com uma inserção conjuntiva longa, não se compromete a longevidade do periodonto de proteção dos dentes de ancoragem. 


\title{
Computed tomography evaluation of rapid maxillary expansion: a case report
}

\begin{abstract}
This paper presents a computed tomography (CT) evaluation of rapid maxillary expansion dentoskeletal and periodontal effects, in a 11.6 year-old girl presenting Class I malocclusion with posterior unilateral crossbite. The toothborne expander with Hyrax screw was activated 7-mm. The patient was submitted to helicoidal CT scan before expansion and after the three-month retention period when the expander was removed. Axial sections with 1-mm thickness were performed parallel to the palatal plane, comprising dentoalveolar and basal areas of maxilla, up to the inferior third of the nasal cavity. Multiplanar reconstruction was employed for achievement of the following measurements by means of the computerized method: maxillary transverse dimensions, posterior teeth inclination, buccal and lingual bone plates thickness, and buccal alveolar crest level. The rapid maxillary expansion yielded a significant transverse increase in all areas measured, of decreasing magnitude from dental arch to basal bone. Posterior teeth were moved in buccal direction, which was associated to both inclination and translation. This orthodontic effect produced a buccal bone plate thickness redution and a lingual bone plate thickness increase. After expansion, bone dehiscences were observed on supporting teeth buccal aspect.
\end{abstract}

Key words: Rapid maxillary expansion. Tomography. X-ray computed.

\section{REFERÊNCIAS}

1. ADKINS, M. D.; NANDA, R. S.; CURRIER, G. F. Arch perimeter changes on rapid palatal expansion. Am J Orthod Dentofacial Orthop, St. Louis, v. 97, no. 3, p.194-199, Mar. 1990.

2. ANDLIN-SOBOCKI, A.; PERSSON, M. The association between spontaneous reversal of gingival recession in mandibular incisors and dentofacial changes in children. A 3-year longitudinal study. Eur J Orthod, London, v.16, no. 3, p. 229-239, June 1994.

3. ÅRTUN, J.; GROBÉTY, D. Periodontal status of mandibular incisors after pronounced orthodontic advancement during adolescence: a follow-up evaluation. Am J Orthod Dentofacial Orthop, St. Louis, v.119, no. 1, p. 2-10, Jan. 2001

4. ÅRTUN, J.; KROGSTAD, O. Periodontal status of mandibular incisors following excessive proclination. A study in adults with surgically treated mandibular prognatism. Am J Orthod Dentofacial Orthop, St. Louis, v. 91, no. 3, p. 225-232, Mar. 1987.

5. BISHARA, S. E.; STALEY, R. N. Maxillary expansion: clinical implications. Am J Orthod Dentofacial Orthop, St. Louis, v. 91 no. 1, p. 3-14, Jan. 1987.

6. BROOKS, S. L. Computed tomography. Dent Clin North Am, Philadelphia, v. 37, no. 4, p. 575-590, Oct. 1993.

7. CAPELOZZA FILHO, L.; SILVA FILHO, O.G. Expansão rápida da maxila: considerações gerais e aplicação clínica. Parte II. R Dental Press Ortodon Ortop Facial, Maringá, v. 2, n. 4, p. 86-108, jul./ago. 1997.

8. CAVALCANTI, M. G. P.; VANNIER, M. W. Measurement of the volume of oral tumors by three-dimensional spiral computed tomography. Dentomaxillofac Radiol, Tokyo, v. 29, no. 1, p. 35-40, Jan. 2000.

9. CAVALCANTI, M. G. P.; RUPRECHT, A.; VANNIER, M. W. Evaluation of an ossifying fibroma using three-dimensional computed tomography. Dentomaxillofac Radiol, Tokyo, v. 30, no. 6, p. 342-345, Nov. 2001.

10. CLEALL, J. F. et al. Expansion of the midpalatal suture in the monkey. Angle Orthod, Appleton, v. 35, no. 1, p. 23-35, Jan. 1965.
11. EKESTUBBE, A. et al. Absorbed doses from computed tomography for dental implant surgery: comparison with conventional tomography. Dentomaxillofac Radiol, Tokyo, v. 22, no. 1, p.13-17, Feb. 1993

12. ERICSON, S.; KUROL, J. CT diagnosis of ectopically maxillary canines- a case report. Eur J Orthod, London, v.10, no. 2, p.115-121, May 1988.

13. ERICSON, S.; KUROL, J. Incisor root resorptions due to ectopic maxillary canines imaged by computerized tomography: a comparative study in extracted teeth. Angle Orthod, Appleton, v. 70, no. 4, p. 276-283, Aug. 2000.

14. ERICSON, S.; BJERKLIN, K.; FALAHAT, B. Does the canine dental follicle cause resorption of permanent incisor roots? A computed tomographic study of erupting maxillary canines. Angle Orthod, Appleton, v. 72, no. 2, p. 95-104, Apr. 2002.

15. FLOYD, P.; PALMER, P.; PALMER, R. Radiographic techniques. Br Dent J, London, v. 187, no. 7, p. 359-365, Oct. 1999.

16. FREDERIKSEN, N.L. Specialized radiographic techniques. In: GOAZ, P. W.; WHITE, S. C. Oral radiology: principles and interpretation. 3rd ed. St. Louis: C. V. Mosby, 1994. cap.13, p. 266-290.

17. FUHRMANN, R. A. W.; BÜCKER, A.; DIEDRICH, P. R. Assessment of alveolar bone loss with high resolution computed tomography. J Periodontal Res, Copenhagen, v. 30, p. 258-263, 1995.

18. FUHRMANN, R. A. W. et al. Assessment of the dentate alveolar process with high resolution computed tomography. Dentomaxillofac Radiol, Tokyo, v. 24, no.1, p. 50-54, Feb. 1995.

19. GREENBAUM, K. R.; ZACHRISSON, B. U. The effect of palatal expansion therapy on the periodontal supporting tissues. Am J Orthod, St. Louis, v. 81, no. 1, p. 12-21, Jan. 1982.

20. HAAS, A. J. Rapid expansion of the maxillary dental arch and nasal cavity by opening the midpalatal suture. Angle Orthod, Appleton, v. 31, no. 2, p. 73-90, Apr. 1961.

21. HAAS, A. J. The treatment of maxillary deficiency by opening the midpalatal suture. Angle Orthod, Appleton, v. 5, no. 3, p. 200-217, July 1965.

22. HOUNSFIELD, G. N. Computed transverse axial scanning (tomography): Part I. Description of system. Br J Radiol, London, v. 46, no. 552, p.1016-1022, Dec. 1973. 
23. ISAACSON, R. J.; MURPHY, T. D. Some effects of rapid maxillary expansion in cleft lip and palate patients. Angle Orthod, Appleton, v. 34, no. 3, p.143-154, July 1964.

24. KREBS, A. Expansion on the midpalatal suture, studied by means of metallic implants. Acta Odontol Scand, Stockholm, v. 92, no. 5, p. 491-501, Dec. 1959.

25. KREBS, A. Midpalatal suture expansion studied by the implant method over a seven-year period. Eur Orthod Soc, London, v. 40, p.131-142, July 1964.

26. LANGLAIS, R. P.; LANGLAND, O. E.; NORTJÉ, C. J. Decision making in dental radiology. In:_. Diagnostic imaging of the jaws. Baltimore: Williams \& Wilkins, 1995. cap.1, p.1-17.

27. LECOMBER, A. R. et al. Comparison of patient dose from imaging protocols for dental implant planning using conventional radiography and computed tomography. Dentomaxillofac Radiol, Tokyo, v. 30, no. 5, p. 255-259, Sept. 2001

28. McNAMARA JR., J. A. Maxillary transverse deficiency. Am J Orthod Dentofacial Orthop, St. Louis, v. 117, no. 5, p. 567-570, May 2000.

29. MELSEN, B. Limitations in adult orthodontics. In: Current controversies in orthodontics. Chicago: Quintessence, 1991. cap.7, p.147-79

30. PAPAIZ, E. G.; CARVALHO, P. L. Métodos recentes de diagnóstico através da imagem. In: FREITAS, A.; ROSA, J. E.; FARIA E SOUZA, I. Radiologia odontológica. 4. ed. São Paulo: Artes Médicas, 1998. cap. 34, p. 667-684.

31. PARKS, E. T. Computed tomography applications for dentistry. Dent Clin North Am, Philadelphia, v. 44, no. 2, p. 371-394, Apr. 2000.

32. PRATO, G. P. et al. Mucogengival interceptive surgery of bucallyerupted premolars in patients scheduled for orthodontic treatment. II. Surgically treated versus nonsurgically treated cases. J Periodontol, Chicago, v. 71, no. 2, p.182-187, Feb. 2000.

33. PREDA, L. et al. The use of spiral computed tomography in the localization of impacted maxillary canines. Dentomaxillofac Radiol, Tokyo, v. 26, no. 4, p. 236-241, July 1997.

34. ROMANELLI, H.; ADAMS, E. Cirugía plástica periodontal. Ubicación en el tratamiento ortodóntico y sus posibilidades. In: HARFIN, J. R. Tratamiento ortodóntico en el adulto. Buenos Aires: Medica Panamericana, 1999. cap.13, p. 485-515.

35. SANTOS, D. T. et al. Aplicação da 2D-TC para diagnóstico e planejamento cirúrgico de lesões fibro-ósseas. Rev Ass Paul Cirurg Dent, São Paulo, v. 57, n. 3, p. 185-190, maio/jun. 2003.

36. SÄRNAS, K. V.; BJÖRK, A.; RUNE, B. Long-term effect of rapid maxillary expansion studied in one patient with the aid of metallic implants and roentgen stereometry. Eur J Orthod, London, v. 14, no. 6, p. 427-432, Dec. 1992

37. SCHMUTH, G. P. F. et al. The application of computerized tomography (CT) in cases of impacted maxillary canines. Eur $\mathbf{J}$ Orthod, London, v. 14, no. 4, p. 296-301, Aug. 1992

38. SILVA FILHO, O. G. da; PRADO MONTES, L. A.; TORELLY, L. F. Rapid maxillary expansion in the deciduous and mixed dentition evaluated through posteroanterior cephalometric analysis. Am
J Orthod Dentofacial Orthop, St. Louis, v. 107, no. 3, p. 268275, Mar. 1995

39. SILVA FILHO, O. G. da; VILLAS BOAS, M. C.; CAPELOZZA FILHO, L. Rapid maxillary expansion in the primary and mixed dentitions: a cephalometric evaluation. Am J Orthod Dentofacial Orthop, St. Louis, v. 100, no. 2, p.171-179, Aug. 1991

40. SIQUEIRA, D. F.; ALMEIDA, R. R.; HENRIQUES, J. F. C. Estudo comparativo, por meio de análise cefalométrica em norma frontal, dos efeitos dentoesqueléticos produzidos por três tipos de expansores palatinos. R Dental Press Ortodon Ortop Facial, Maringá, v. 7, n. 6, p. 27-47, nov./dez. 2002.

41. SMITH, R. J.; BURSTONE, C. J. Mechanics of tooth movement Am J Orthod Dentofacial Orthop, St. Louis, v. 85, no. 4 p. 294-307, Apr. 1984.

42. STARNBACH, $\mathrm{H}$. et al. Facioskeletal and dental changes resulting from rapid maxillary expansion. Angle Orthod, Appleton, v. 36, no. 2, p. 152-164, Apr. 1966.

43. STEINER, G. G.; PEARSON, J. K.: AINAMO, J. Changes of the marginal periodontium as a result of labial tooth movement in monkeys. J Periodontol, Chicago, v. 52, no. 6, p. 314-320 June 1981.

44. TANRIKULU, R.; EROL, B. Comparison of computed tomography with conventional radiography for midfacial fractures. Dentomaxillofac Radiol, Tokyo, v. 30, no. 3, p. 141-146, May 2001.

45. THILANDER, B. et al. Bone regeneration in alveolar bone dehiscences related to orthodontic tooth movements. Eur J Orthod, London, v. 5, no. 2, p.105-114, May 1983.

46. VAN DER STELT, P. F.; DUNN, S. M. 3D-Imaging in dental radiology. In: FARMAN, A.G. et al. Advances in maxillofacial imaging. Amsterdam: Elsevier, 1997. p. 367-372.

47. VISSER, H. et al. Doses to critical organs from computed tomography (CT). In: FARMAN, A. G. et al. Advances in maxillofacial imaging. Amsterdam: Elsevier, 1997. p. 401-406.

48. VITRAL, R. W. F.; TELLES, C. S. T. Computed tomography evaluation of temporomandibular joint alterations in Class II division 1 subdivision patients: condylar symmetry. Am J Orthod Dentofacial Orthop, St. Louis, v. 121, no. 4, p. 369-375, Apr. 2002.

49. WAGENBERG, B. D. Periodontal preparation of the adult patient prior to orthodontics. Dent Clin North Am, Philadelphia, v. 32, no. 3, p. 457-480, July 1988.

50. WENNSTRÖM, J. L.; PRATO, G. P. P. Terapia mucogengival. In: LINDHE, J. Tratado de periodontia clínica e implantologia oral. 3. ed. Rio de Janeiro: Guanabara Koogan, 1999. cap.19, p. 393-427.

51. WENNSTRÖM, J. L. et al. Some periodontal tissue reactions to orthodontic tooth movement in monkeys. J Clin Periodontol, Chicago, v.14, no. 3, p.121-129, Mar. 1987.

52. WERTZ, R. A. Skeletal and dental changes accompanying rapid midpalatal suture opening. Am J Orthod, St. Louis, v. 58, no. 1 p. 41-66, July 1970.

53. ZACHRISSON, B.U. Ortodontia e periodontia. In: LINDHE, J. Tratado de periodontia clínica e implantologia oral. 3. ed. Rio de Janeiro: Guanabara Koogan, 1999. cap. 25, p. 537-580.
Endereço para correspondência

Daniela G. Garib

R. Rio Branco n. 19-18

Bauru - SP CEP 17040-480

e-mail: dgarib@uol.com.br 